Pacific

Journal of

Mathematics

ON THE $(\mathfrak{g}, K)$-COHOMOLOGY OF CERTAIN THETA LIFTS

Soo Teck Lee And Chen-Bo Zhu

Volume 199 No. 1

May 2001 


\title{
ON THE $(\mathfrak{g}, K)$-COHOMOLOGY OF CERTAIN THETA LIFTS
}

\author{
Soo Teck Lee And Chen-Bo Zhu
}

\begin{abstract}
Let $\theta^{p, q}$ be the theta lift of $S p(4, \mathbb{R})$ from the trivial representation of $O(p, q)$, where $p+q$ is even. We compute the $(\mathfrak{g}, K)$-cohomology of $\theta^{p, q}$ by embedding them into certain degenerate principal series representations of $S p(4, \mathbb{R})$.
\end{abstract}

\section{Introduction and main result.}

It is well-known that one of the most important ways of constructing cohomology for an arithmetic quotient $\Gamma \backslash X$, where $X$ is the symmetric space associated with a semi-simple Lie group $G$ with finite center and $\Gamma$ is a discrete subgroup of $G$, is through the use of Matsushima's formula, namely by exhibiting irreducible unitary representations with non-zero continuous cohomology which occur in $L^{2}(\Gamma \backslash G)$. (See $[\mathbf{B W}]$.) In this connection, the machinery of theta correspondence plays a major role, and the technique of lifting a discrete series representation of $G^{\prime}$, where $\left(G^{\prime}, G\right)$ forms a dual pair, proves to be an especially powerful tool. The basis of such applications lies in the fact that when $G^{\prime}$ is relatively small compared to $G$, a sufficiently regular discrete series representation of $G^{\prime}$ will be lifted to a unitary representation of $G$ with non-zero cohomology ([RS], $[\mathbf{A d}],[\mathbf{L i 2}])$. Examples of such applications were given by Kazhdan [Ka] (from $U(1)$ to $S U(n, 1)$ ), Borel-Wallach [BW] (from $U(1)$ to $S U(p, q)$ ), Anderson [An] (with $G^{\prime}$ compact), and in general by $\mathrm{Li}$ for reductive dual pairs of type 1 [Li3]. We note that all the representations of $G^{\prime} \times G$ involved in these applications are in the discrete spectrum of the restriction of the oscillator representation, and are automatically unitary.

It is an interesting question to investigate the cohomological properties of the representations which arise in the formalism of theta correspondence and which do not (necessarily) belong to the discrete spectrum. The most interesting examples should be theta lifts of one dimensional representations, and there is reason to believe that these representations often have nonzero cohomology when the requirement for infinitesimal character is met (cf. Wigner's Lemma, $[\mathbf{B W}]$ ). In this article, we will be concerned with the continuous cohomology of (not necessarily unitary) representations of 
$S p(2 n, \mathbb{R})$ which are the theta lift of the trivial representation of $O(p, q)$. We will determine their continuous cohomology completely in the case $n=2$.

To be more precise, let $\left(G^{\prime}, G\right)=(O(p, q), S p(2 n, \mathbb{R}))$ be the reductive dual pair in $S p=S p(2 n(p+q), \mathbb{R})$. Let $\widetilde{S} p$ be its two-fold (metaplectic) cover. Fix an oscillator representation $\omega$ of $\widetilde{S} p[\mathbf{H 2}]$. Let $\mathcal{Y}$ denote the Hilbert space on which $\omega$ acts, and let $\mathcal{Y}^{\infty} \subset \mathcal{Y}$ be the subspace of smooth vectors for $\widetilde{S} p$. Assume that $m=p+q$ is even. Let $\Omega^{p, q}$ be the maximal quotient of $\mathcal{Y}^{\infty}$ on which $O(p, q)$ acts trivially. Following Kudla and Rallis [KR1], we call $\Omega^{p, q}$ the Howe quotient corresponding to the trivial representation of $O(p, q)$. Then from Howe's classical work, we know that $\Omega^{p, q}$ is a quasi-simple admissible representation of $S p(2 n, \mathbb{R})$, and it has a unique irreducible quotient $\theta^{p, q}([\mathbf{H} 2])$. Thus $\theta^{p, q}$ is the representation of $S p(2 n, \mathbb{R})$ corresponding to the trivial representation of $O(p, q)$ under Howe's quotient correspondence, and is usually called the theta lift (or the Howe lift) of the trivial representation. The study of these representations was initiated by Kudla and Rallis [KR1] in relation to degenerate principal series and invariant distributions. The global versions of such representations play a very important role in the celebrated Siegel-Weil formula and its extentions [KR2]. The structure of $\theta^{p, q}$ (and $\Omega^{p, q}$ ) has been completely determined by the authors in [LZ2]. We also remark that the set of $\theta^{p, q}$ for $p+q \leq n$ is precisely the set of unipotent representations of $S p(2 n, \mathbb{R})$ constructed by Sahi $[\mathbf{S}]$.

We shall now fix some notation. Let $\mathfrak{g}=\mathfrak{s p}(2 n, \mathbb{C})$ be the complexified Lie algebra of $S p(2 n, \mathbb{R})$. Let $I_{n}$ denote the $n \times n$ identity matrix, and let

$$
K=\left\{\left(\begin{array}{cc}
A & B \\
-B & A
\end{array}\right): A, B \in G L(n, \mathbb{R}), A B^{t}=B^{t} A, A A^{t}+B B^{t}=I_{n}\right\} .
$$

Then $K \cong U(n)$ and is a maximal compact subgroup of $S p(2 n, \mathbb{R})$. Thus the weights of $K$ can be parametrized in a standard way by $n$-tuples of integers $\left(\lambda_{1}, \ldots, \lambda_{n}\right)$ (see $\left.[\mathbf{B T}]\right)$. In particular, the set of dominant weights of $K$ can be identified with the set

$$
\Lambda^{+}(n)=\left\{\lambda=\left(\lambda_{1}, \ldots, \lambda_{n}\right) \in \mathbb{Z}^{n}: \lambda_{1} \geq \cdots \geq \lambda_{n}\right\}
$$

For each $\lambda \in \Lambda^{+}(n)$ such that $\lambda_{n} \geq 0$, let $F_{\lambda}$ denote the finite dimensional irreducible representation of $G$ with the highest weight $\lambda$.

The following proposition summaries some basic properties of $\theta^{p, q}$. 


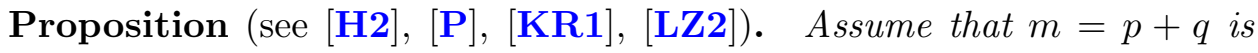
even. Denote $\mathbf{1}_{n}=\underbrace{(1, \ldots, 1)}_{n}$.

(a) $\theta^{p, q}$ has the infinitesimal character with Harish-Chandra parameter $\left(\frac{m}{2}-1, \frac{m}{2}-2, \ldots, \frac{m}{2}-n\right)$.

(b) $\theta^{p, q}$ is finite dimensional if and only if $p, q \equiv n+1(\bmod 2)$, and $p, q \geq n+1$. In this case, $\theta^{p, q}$ is isomorphic to $F_{\left(\frac{m}{2}-n-1\right) \mathbf{1}_{n}}$.

(c) $\theta^{m, 0}$ (resp. $\left.\theta^{0, m}\right)$ is the unitary lowest (resp. highest) weight representation with lowest weight $\frac{m}{2} \mathbf{1}_{n}$ (resp. highest weight $-\frac{m}{2} \mathbf{1}_{n}$ ).

(d) $\theta^{p, q}$ is unitary if and only if either $p q=0$ or both $p, q \leq n+1$.

To avoid too many notations, we shall not distinguish a smooth representation from the corresponding $(\mathfrak{g}, K)$-module. In the same way we shall not distinguish the continuous cohomology of a smooth representation from the $(\mathfrak{g}, K)$-cohomology of the underlying Harish-Chandra module. We now state a result which follows easily from the above proposition. As usual $H^{i}(\mathfrak{g}, K ; \pi)$ denotes the $i$ th $(\mathfrak{g}, K)$-cohomology group of a finitely generated admissible $(\mathfrak{g}, K)$-module $\pi([\mathbf{B W}])$.

We shall now restrict ourselves to the case $n=2$.

Proposition. Assume that $n=2$ and $m=p+q$ is even.

(a) If $F$ is any finite dimensional irreducible representation of $G$ and $m \leq$ 4 , then $H^{*}\left(\mathfrak{g}, K ; \theta^{p, q} \otimes F^{*}\right)=0$.

(b) Suppose that $m \geq 6$ and $p, q \geq 3$ are both odd. Then $\theta^{p, q} \cong F_{\left(\frac{m}{2}-3, \frac{m}{2}-3\right)}$, so that

$$
H^{i}\left(\mathfrak{g}, K ; \theta^{p, q} \otimes F_{\left(\frac{m}{2}-3, \frac{m}{2}-3\right)}^{*}\right)= \begin{cases}\mathbb{C}, & i=0,2,4,6 \\ 0, & i=1,3,5\end{cases}
$$

(c) Suppose that $m \geq 6$. Then $\theta^{m, 0}$ (resp. $\theta^{0, m}$ ) is a holomorphic (resp. anti-holomorphic) discrete series representation of $S p(4, \mathbb{R})$, so that

$$
\begin{aligned}
H^{i}\left(\mathfrak{g}, K ; \theta^{m, 0} \otimes F_{\left(\frac{m}{2}-3, \frac{m}{2}-3\right)}^{*}\right) & =H^{i}\left(\mathfrak{g}, K ; \theta^{0, m} \otimes F_{\left(\frac{m}{2}-3, \frac{m}{2}-3\right)}^{*}\right) \\
& = \begin{cases}\mathbb{C}, & i=3, \\
0, & i=0,1,2,4,5,6 .\end{cases}
\end{aligned}
$$

Our main result is a computation of the $(\mathfrak{g}, K)$-cohomology of $\theta^{p, q}$ in all the other cases.

Main Theorem. Assume that $n=2$ and $m=p+q$ is even. 
(a) Suppose that $m \geq 6$. Then

$$
\begin{aligned}
H^{i}\left(\mathfrak{g}, K ; \theta^{m-1,1} \otimes F_{\left(\frac{m}{2}-3, \frac{m}{2}-3\right)}^{*}\right) & =H^{i}\left(\mathfrak{g}, K ; \theta^{1, m-1} \otimes F_{\left(\frac{m}{2}-3, \frac{m}{2}-3\right)}^{*}\right) \\
& = \begin{cases}\mathbb{C}, & i=1,3,5 \\
0, & i=0,2,4,6 .\end{cases}
\end{aligned}
$$

(b) Suppose that $m \geq 6$ and $p, q \geq 2$ are both even. Then

$$
H^{i}\left(\mathfrak{g}, K ; \theta^{p, q} \otimes F_{\left(\frac{m}{2}-3, \frac{m}{2}-3\right)}^{*}\right)= \begin{cases}\mathbb{C}, & i=2,4 \\ 0, & i=0,1,3,5,6\end{cases}
$$

Thus in the case $n=2, \theta^{p, q}$ either has zero $(\mathfrak{g}, K)$-cohomology for an obvious reason, namely its infinitesimal character does not match that of any finite dimensional representation, or has regular patterns of cohomology. It will be very interesting to examine to what extent this phenomenon persists for general $n$, and to investigate its connection (if any) to cohomology in geometric settings alluded to in the begining of the introduction. In this connection, we mention the work of Wallach $[\mathbf{W}]$ which studies such questions for $G=S U(p, q)$.

We finally note that irreducible unitary representations with non-zero continuous cohomology were classified by Vogan and Zuckerman [VZ], and are all cohomologically induced from unitary characters of Levi subgroups (associated to $\theta$ stable parabolic subalgebras). With the exception of $\theta^{m, 0}$, $\theta^{0, m}(m \geq 6)$ which are discrete series, all the other $\theta^{p, q}$ whose $(\mathfrak{g}, K)$ cohomology we have computed do not fall in this list, since they are not unitary.

This paper is arranged as follows. In Section 2, we compute the $(\mathfrak{g}, K)$ cohomology of the degenerate principal series $I^{ \pm}(\sigma)$ of $S p(4, \mathbb{R})$ studied in [Le2]. We compute the $(\mathfrak{g}, K)$-cohomology of the irreducible constituents of $I^{ \pm}(\sigma)$ in Section 3. As the theta lift can be identified with some of these constituents $[\mathbf{L Z 2}]$, our main theorem follows.

We would like to thank Professors Roger Howe and Michael Harris for posing a related question on secondary invariants in cohomology, which provides the initial impetus to this project. 


\section{2. $(\mathfrak{g}, K)$-cohomology of the degenerate series.}

Let $G=S p(2 n, \mathbb{R})$ and let $P=M N$ be the Siegel parabolic subgroup of $G$ where

$$
\begin{aligned}
M & =\left\{m_{a}=\left(\begin{array}{cc}
a & 0 \\
0 & a^{-1}
\end{array}\right): a \in G L_{n}(\mathbb{R})\right\}, \\
N & =\left\{n_{b}=\left(\begin{array}{ll}
1 & b \\
0 & 1
\end{array}\right): b \in M_{n}(\mathbb{R}), b^{t}=b\right\} .
\end{aligned}
$$

For each $\sigma \in \mathbb{C}$, we let $\chi_{\sigma}^{ \pm}: P \mapsto \mathbb{C}$ be the characters given by

$$
\chi_{\sigma}^{ \pm}\left(m_{a} n_{b}\right)= \begin{cases}(\operatorname{det} a)^{\sigma} & \text { if } \operatorname{det} a>0 \\ \pm|\operatorname{det} a|^{\sigma} & \text { if } \operatorname{det} a<0\end{cases}
$$

and let $I^{ \pm}(\sigma)$ be the corresponding (normalized) induced representations. The representation spaces for $I^{ \pm}(\sigma)$ are respectively

$$
\left\{f \in C^{\infty}(S p(2 n, \mathbb{R})): f(p g)=\delta(p)^{\frac{1}{2}} \chi_{\sigma}^{ \pm}(p) f(p), g \in S p(2 n, \mathbb{R}), p \in P\right\},
$$

where $\delta$ is the modular function of $P$ given by $\delta\left(m_{a} n_{b}\right)=|\operatorname{det} a|^{n+1}$, and $S p(2 n, \mathbb{R})$ acts on the representation spaces by right translation. The module structure of these representations have been studied by the first-named author in $[\mathbf{L e} 2]$. In this section we shall compute the $(\mathfrak{g}, K)$ cohomology of $I^{ \pm}(\sigma)$ in the case $n=2$ and when it is reducible. Our computation follows closely $[\mathbf{B W}]$ and $[\mathbf{Z}]$.

For the moment we assume that $n \geq 2$. By the result of $[\mathbf{K R} \mathbf{R}], I^{ \pm}(\sigma)$ is reducible if and only if $\sigma \in \frac{n+1}{2}+\mathbb{Z}$. Thus, in this case we can write $\sigma=\frac{m-(n+1)}{2}$, where $m$ is an even integer.

Given a finitely generated admissible $(\mathfrak{g}, K)$-module $\pi$ and a finite dimensional representation $F$ of $G$, it is well-known $[\mathbf{B W}]$ that $H^{i}\left(\mathfrak{g}, K ; \pi \otimes F^{*}\right)$ is canonically isomorphic to the dual of $H^{l-i}(\mathfrak{g}, K ; \tilde{\pi} \otimes F)$, where $l=\operatorname{dim} \mathfrak{g} / \mathfrak{k}=$ $n(n+1)$, and $\tilde{\pi}$ is the $K$-finite dual of $\pi$. Since the Harish-Chandra modules of $I^{ \pm}(\sigma)$ and $I^{ \pm}(-\sigma)$ are $K$-finite duals of each other, we may restrict our attention to the case where $\sigma \geq 0$, namely $m \geq n+1$.

Recall that we denote by $F_{\lambda}$ the finite dimensional irreducible representation of $G$ with the highest weight $\lambda=\left(\lambda_{1}, \ldots, \lambda_{n}\right)$, where $\lambda_{1} \geq \cdots \geq \lambda_{n} \geq 0$ are integers.

Lemma 1. We have

$$
H^{i}\left(\mathfrak{g}, K ; I^{ \pm}\left(\frac{m-(n+1)}{2}\right) \otimes F_{\lambda}^{*}\right)=0
$$

unless $m \geq 2 n+2$, and $\lambda=\left(\frac{m}{2}-(n+1)\right) 1_{n}$. Here $1_{n}=(1, \ldots, 1)$. 
Proof. An easy computation implies that the infinitesimal character of $I^{ \pm}\left(\frac{m-(n+1)}{2}\right)$ has the Harish-Chandra parameter $\left(\frac{m}{2}-1, \frac{m}{2}-2, \ldots, \frac{m}{2}-n\right)$. From Wigner's Lemma $[\mathbf{B W}], H^{i}\left(\mathfrak{g}, K ; I^{ \pm}\left(\frac{m-(n+1)}{2}\right) \otimes F_{\lambda}^{*}\right)=0$ unless the infinitesimal characters of $I^{ \pm}\left(\frac{m-(n+1)}{2}\right)$ and $F_{\lambda}$ are the same. Now the infinitesimal character of $F_{\lambda}$ has the Harish-Chandra parameter $\lambda+\rho$, where $\rho=(n, n-1, \cdots, 1)$. The lemma then follows immediately.

Let $s g n$ be the signum character of $M \cong G L(n, \mathbb{R})$ :

$$
\operatorname{sgn}\left(m_{a}\right)= \begin{cases}1 & \text { if } \operatorname{det} a>0 \\ -1 & \text { if } \operatorname{det} a<0\end{cases}
$$

where $m_{a}=\left(\begin{array}{cc}a & 0 \\ 0 & a^{-1}\end{array}\right)$ and $a \in G L(n, \mathbb{R})$. We shall also denote the restriction of sgn to $M \cap K \cong O(n)$ by sgn.

Proposition 1. Let $m \geq 2 n+2$ be an even integer. Then for $0 \leq i \leq$ $\frac{n(n+1)}{2}$,

$$
H^{i}\left(\mathfrak{g}, K ; I^{\epsilon}\left(\frac{m-(n+1)}{2}\right) \otimes F_{\left(\frac{m}{2}-(n+1)\right) 1_{n}}^{*}\right)=0,
$$

and for $\frac{n(n+1)}{2} \leq i \leq n(n+1)$,

$H^{i}\left(\mathfrak{g}, K ; I^{\epsilon}\left(\frac{m-(n+1)}{2}\right) \otimes F_{\left(\frac{m}{2}-(n+1)\right) 1_{n}}^{*}\right) \cong\left(\Lambda^{i-\frac{n(n+1)}{2}}\left(S^{2}\left(\mathbb{C}^{n}\right)\right)^{*}\right)_{O(n)}^{\chi}$, where $\chi$ is the character of $O(n)$ given by

$$
\chi= \begin{cases}\text { trivial } & \text { if } \epsilon=+, m \equiv 0(\bmod 4), \text { or } \epsilon=-, m \equiv 2(\bmod 4), \\ \operatorname{sgn} & \text { if } \epsilon=+, m \equiv 2(\bmod 4), \text { or } \epsilon=-, m \equiv 0(\bmod 4),\end{cases}
$$

$\Lambda^{i}\left(S^{2}\left(\mathbb{C}^{n}\right)\right)$ is the ith exterior power of the symmetric square of $\mathbb{C}^{n}$, and $\left(\Lambda^{i}\left(S^{2}\left(\mathbb{C}^{n}\right)\right)^{*}\right)_{O_{n}}^{\chi}$ is the space of relative invariants of the dual of $\Lambda^{i}\left(S^{2}\left(\mathbb{C}^{n}\right)\right)$ with respect to the character $\chi$.

Proof. Let $\lambda=\left(\frac{m}{2}-(n+1)\right) 1_{n}$ and write $I^{ \pm}$for $I^{ \pm}\left(\frac{m-(n+1)}{2}\right)$. Note that $F_{\lambda}^{*} \cong F_{\lambda}$.

Following Borel and Wallach ([BW, Chapter 3]), we have for $1 \leq i \leq$ $n(n+1)$,

$$
H^{i}\left(\mathfrak{g}, K ; I^{ \pm} \otimes F_{\lambda}\right) \cong H^{i}\left(\mathfrak{p}, P \cap K ;\left.\chi_{\frac{m}{2}}^{ \pm} \otimes F_{\lambda}\right|_{P}\right),
$$

where $\mathfrak{p}$ is the complexified Lie algebra of $P$.

There is a Hochschild-Serre spectral sequence $\left(E_{r}\right)$ abutting to $H^{*}(\mathfrak{p}, P \cap$ $\left.K ;\left.\chi_{\frac{m}{2}}^{ \pm} \otimes F_{\lambda}\right|_{P}\right)$ in which the $E_{2}$-term is

$$
E_{2}^{p, q}=H^{p}\left(\mathfrak{m}, M \cap K ;\left.H^{q}\left(\mathfrak{n} ; F_{\lambda}\right) \otimes \chi_{\frac{m}{2}}^{ \pm}\right|_{M}\right) .
$$


(See $[\mathbf{B W}]$.$) Here \mathfrak{m} \cong \mathfrak{g l}_{n}(\mathbb{C})$ is the complexified Lie algebra of $M$.

Let $W$ and $W_{M}$ be the Weyl groups of $G$ and $M$, respectively. Then Kostant's formula [Ko] yields

$$
H^{q}\left(\mathfrak{n} ; F_{\lambda}\right)=\oplus_{s \in W^{1}, l(s)=q} L_{s(\lambda+\rho)-\rho},
$$

where $W^{1}=\left\{w \in W \mid w^{-1}(\alpha)>0\right.$ for $\left.\alpha \in \Delta_{M}\right\}$ is a set of representatives for the right cosets of $W_{M}$ in $W$, and for $s \in W^{1}, L_{s(\lambda+\rho)-\rho}$ denotes the finite dimensional irreducible representation of $M$ with highest weight $s(\lambda+\rho)-\rho$, and $l(s)$ is the length of $s$. It follows that

$$
E_{2}^{p, q}=\oplus_{s \in W^{1}, l(s)=q} H^{p}\left(\mathfrak{m}, M \cap K ;\left.L_{s(\lambda+\rho)-\rho} \otimes \chi_{\frac{m}{2}}^{ \pm}\right|_{M}\right) .
$$

Another application of Wigner's lemma implies that

$$
H^{p}\left(\mathfrak{m}, M \cap K ;\left.L_{s(\lambda+\rho)-\rho} \otimes \chi_{\frac{m}{2}}^{ \pm}\right|_{M}\right)=0
$$

unless the infinitesimal character of $L_{s(\lambda+\rho)-\rho}$ is equal to the infinitesimal character of $\chi_{-\frac{m}{2}}^{ \pm}$as a $M$ module.

Let $\mathfrak{h} \cong \mathbb{C}^{n}$ be the standard Cartan subalgebra consisting of diagonal elements in $\mathfrak{m} \subseteq \mathfrak{g}$. Let $A$ be the split component of $M$. Then we have

$$
\begin{aligned}
s(\lambda+\rho)-\left.\rho\right|_{A} & =\left.\chi_{\frac{m}{2}}^{\mp}\right|_{A}, \\
s(\lambda+\rho)-\left.\rho\right|_{\mathfrak{h}} & =\left.\chi_{\frac{m}{2}}^{\mp}\right|_{\mathfrak{h}} .
\end{aligned}
$$

Since $\lambda+\rho$ is regular, $s$ is fixed uniquely by the above conditions.

By a straight-forward calculation we see that $s$ is the element of $W^{1}$ which sends $\left(\nu_{1}, \cdots, \nu_{n}\right) \in \mathfrak{h}$ to $\left(-\nu_{n}, \cdots,-\nu_{1}\right)$. Note that $l(s)=\frac{n(n+1)}{2}$ and $s(\lambda+\rho)-\rho=-\frac{m}{2} 1_{n}$. Thus the only possible non-zero term of $E_{2}^{p, q}$ in Eq. (2) comes from this particular $s$, and we have $E_{2}^{p, q}=H^{p}(\mathfrak{m}, M \cap$ $\left.K ;\left.L_{-\frac{m}{2} 1_{n}} \otimes \chi_{\frac{m}{2}}^{ \pm}\right|_{M}\right)$.

Let $\chi$ be the representation of $M$ given by

$$
\chi=\left.L_{-\frac{m}{2} 1_{n}} \otimes \chi_{\frac{m}{2}}^{+}\right|_{M} .
$$

Thus

$$
\chi= \begin{cases}\text { trivial, } & \epsilon=+, m \equiv 0(\bmod 4), \text { or } \epsilon=-, \quad m \equiv 2(\bmod 4), \\ \operatorname{sgn}, & \epsilon=+, m \equiv 2(\bmod 4), \text { or } \epsilon=-, \quad m \equiv 0(\bmod 4) .\end{cases}
$$

It follows that the spectral sequence $\left(E_{r}\right)$ degenerates and we have

$$
H^{i+\frac{n(n+1)}{2}}\left(\mathfrak{g}, K ; I^{ \pm} \otimes F_{\lambda}\right)=E_{2}^{i, \frac{n(n+1)}{2}}=H^{i}(\mathfrak{m}, M \cap K ; \chi) .
$$


Now $H^{i}(\mathfrak{m}, M \cap K ; \chi)=\operatorname{Hom}_{M \cap K}\left(\Lambda^{i}(\mathfrak{m} / \mathfrak{m} \cap \mathfrak{k}), \chi\right)=\left(\Lambda^{i}(\mathfrak{m} / \mathfrak{m} \cap \mathfrak{k})^{*}\right)_{M \cap K}^{\chi}$, the $\chi$-isotypic component of the $i$ th exterior power of $(\mathfrak{m} / \mathfrak{m} \cap \mathfrak{k})^{*}$ considered as a $M \cap K$-module. By identifying $M$ with $G L(n, \mathbb{R})$, we see this is equal to $\left(\Lambda^{i}\left(S^{2}\left(\mathbb{C}^{n}\right)\right)^{*}\right)_{O(n)}^{\chi}$. Hence the proposition follows.

The $G L_{n}$-structure of $\Lambda^{i}\left(S^{2}\left(\mathbb{C}^{n}\right)\right)$ can be described using the result of Kostant $[\mathbf{K o}]$. It turns out that $\Lambda^{i}\left(S^{2}\left(\mathbb{C}^{n}\right)\right)$ is multiplicity-free as a $G L_{n^{-}}$ module, and the young diagram of the constituents are formed by taking nested sequences of hooks of the form $\left(r+1,1^{r-1}\right)$, for a set of integers $r$ (see $[\mathbf{H} 4])$. In the case $n=2$, the $(\mathfrak{g}, K)$-cohomology of $I^{ \pm}\left(\frac{m-3}{2}\right)$ is given in the following proposition.

Proposition 2. Let $n=2$ and let $m \geq 6$ be an even integer. Write $I^{ \pm}=$ $I^{ \pm}\left(\frac{m-3}{2}\right)$ and $F^{*}=F_{\left(\frac{m}{2}-3, \frac{m}{2}-3\right)}^{*}$.

(a) If $m \equiv 2(\bmod 4)$, then

$$
\begin{aligned}
& H^{i}\left(\mathfrak{g}, K ; I^{+} \otimes F^{*}\right)= \begin{cases}\mathbb{C}, & i=5,6, \\
0, & i=0,1,2,3,4 .\end{cases} \\
& H^{i}\left(\mathfrak{g}, K ; I^{-} \otimes F^{*}\right)= \begin{cases}\mathbb{C}, & i=3,4, \\
0, & i=0,1,2,5,6 .\end{cases}
\end{aligned}
$$

(b) If $m \equiv 0(\bmod 4)$, then

$$
\begin{aligned}
& H^{i}\left(\mathfrak{g}, K ; I^{+} \otimes F^{*}\right)= \begin{cases}\mathbb{C}, & i=3,4, \\
0, & i=0,1,2,5,6 .\end{cases} \\
& H^{i}\left(\mathfrak{g}, K ; I^{-} \otimes F^{*}\right)= \begin{cases}\mathbb{C}, & i=5,6, \\
0, & i=0,1,2,3,4 .\end{cases}
\end{aligned}
$$

Proof. We just need to examine the $G L_{2}$-structure of $\Lambda^{i}\left(S^{2}\left(\mathbb{C}^{2}\right)\right)^{*}$. It is multiplicity-free, and the constituents are contragradient to the $G L_{2}$ representations with highest weights $(0,0),(2,0),(3,1),(3,3)$, respectively. They occur in $\Lambda^{i}\left(S^{2}\left(\mathbb{C}^{2}\right)\right)^{*}$ for $i=0,1,2,3$, respectively.

Notice that the first two constituents are in $\left(\Lambda^{i}\left(S^{2}\left(\mathbb{C}^{2}\right)\right)^{*}\right)_{O(2)}^{\chi}$, where $\chi$ is trivial, and the last two constituents are in $\left(\Lambda^{i}\left(S^{2}\left(\mathbb{C}^{2}\right)\right)^{*}\right)_{O(2)}^{\chi}$, where $\chi$ is the signum character. The proposition then follows.

\section{The cohomology of the irreducible constituents.}

Throughout this section, we let $G=S p(4, \mathbb{R}), \mathfrak{g}=\mathfrak{s p}(4, \mathbb{C})$ and $K$ is the maximal compact subgroup of $G$ given in (1) with $n=2$. In this section, we shall compute the $(\mathfrak{g}, K)$-cohomology of the irreducible constituents of $I^{ \pm}\left(\frac{m-3}{2}\right)$. Recall that $\Omega^{p, q}$ is the Howe quotient corresponding to the trivial 
representation of $O(p, q)$ and $\theta^{p, q}$ is the unique irreducible quotient of $\Omega^{p, q}$. From the work of Kudla and Rallis [KR1] (see also [LZ2]), there are $G$ equivariant embeddings

$$
\Omega^{p, q} \hookrightarrow \begin{cases}I^{+}\left(\frac{m-3}{2}\right), & p-q \equiv 0(\bmod 4), \\ I^{-}\left(\frac{m-3}{2}\right), & p-q \equiv 2(\bmod 4),\end{cases}
$$

where $m=p+q$. By the results in $[\mathbf{L Z 2}], \theta^{p, q}$ can be identified with the appropriate irreducible constituents in $I^{ \pm}\left(\frac{m-3}{2}\right)$. Hence our main theorem will follow from results in this section (Theorems 5 and 7 ).

We shall only examine the case of $I^{+}=I^{+}\left(\frac{m-3}{2}\right)$. The case of $I^{-}=$ $I^{-}\left(\frac{m-3}{2}\right)$ is similar.

We write $F=F_{\left(\frac{m}{2}-3, \frac{m}{2}-3\right)}$ and $H^{i}(\pi)=H^{i}(\mathfrak{g}, K ; \pi)$ for any $(\mathfrak{g}, K)$ module $\pi$. We also assume that $m \geq 6$ is and even. There are two cases.

Case 1. $m \equiv 2(\bmod 4)$. In this case, $I^{+}$has a unique irreducible submodule $\pi_{0}$ which is unitary and a unique irreducible quotient which is isomorphic to $F$. In addition, there are two irreducible constituents $\pi_{1,0}$ and $\pi_{0,1}$ (both non-unitary). The module diagram of $I^{+}([\mathbf{L e} 2])$ is given in Figure 1. Here a blackened circle denotes a unitary constituent.

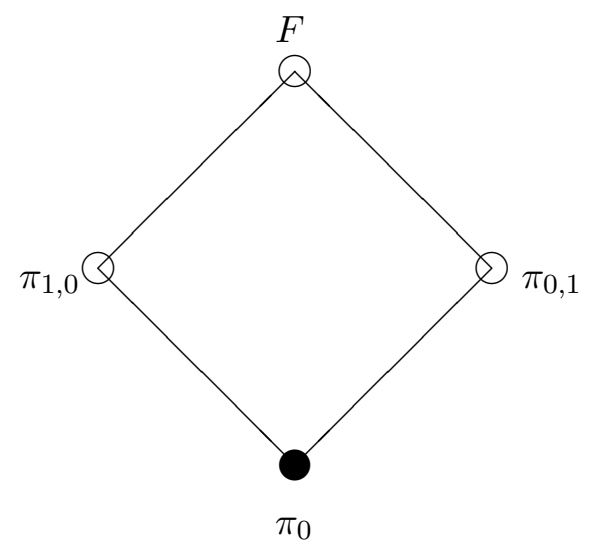

Figure 1.

We know that (see $[\mathbf{L e} 2])$ the highest weights of the $K$-types in $I^{+}\left(\frac{m-3}{2}\right)$ are given by the set

$$
\Lambda_{e}^{+}(2)=\left\{\left(\lambda_{1}, \lambda_{2}\right) \in \Lambda^{+}(2): \lambda_{1} \text { and } \lambda_{2} \text { are even }\right\}
$$


and each $K$-type occurs with multiplicity one. The highest weights of the $K$-types of the irreducible constituents are given in Table 1.

\begin{tabular}{|c|c|}
\hline constituent & highest weights of $K$-types \\
\hline$F$ & $\left(\lambda_{1}, \lambda_{2}\right) \in \Lambda_{e}^{+}(2)$ with $\lambda_{1} \leq \frac{m}{2}-3, \lambda_{2} \geq-\frac{m}{2}+3$ \\
\hline$\pi_{1,0}$ & $\left(\lambda_{1}, \lambda_{2}\right) \in \Lambda_{e}^{+}(2)$ with $\lambda_{1} \geq \frac{m}{2}-1, \lambda_{2} \geq-\frac{m}{2}+3$ \\
\hline$\pi_{0,1}$ & $\left(\lambda_{1}, \lambda_{2}\right) \in \Lambda_{e}^{+}(2)$ with $\lambda_{1} \leq \frac{m}{2}-3, \lambda_{2} \leq-\frac{m}{2}+1$ \\
\hline$\pi_{0}$ & $\left(\lambda_{1}, \lambda_{2}\right) \in \Lambda_{e}^{+}(2)$ with $\lambda_{1} \geq \frac{m}{2}-1, \lambda_{2} \leq-\frac{m}{2}+1$ \\
\hline
\end{tabular}

Table 1.

Proposition 3 ([LZ2]). We have

$$
\theta^{m-1,1} \cong \pi_{1,0}, \quad \theta^{1, m-1} \cong \pi_{0,1} .
$$

Moreover, if both $p$ and $q$ are odd and $p, q \geq 3$, then $\theta^{p, q} \cong F$.

We shall postpone the proof of the following proposition to the end of this section.

Proposition 4. There are vector space isomorphisms $\mathcal{T}_{1}: \pi_{1,0} \rightarrow \pi_{0,1}$ and $\mathcal{T}_{2}: F \rightarrow F$ and a Lie algebra automorphism $\tau$ of $\mathfrak{s p}(4, \mathbb{C})$ of order 2 such that for $i=1,2$,

$$
\mathcal{T}_{i}(X v)=\tau(X) \mathcal{T}_{i}(v)
$$

for all $X \in \mathfrak{s p}(4, \mathbb{C})$ and for all $v$ in the domain of $\mathcal{T}_{i}$.

Recall the unique irreducible quotient $F=F_{\left(\frac{m}{2}-3, \frac{m}{2}-3\right)}$ of $I^{+}$. From Wigner's lemma we know that the $(\mathfrak{g}, K)$-cohomology of an irreducible finite dimensional representation is trivial unless it is the trivial representation. Therefore $H^{i}\left(F \otimes F^{*}\right)=H^{i}$ (trivial) $=\mathbb{C}$ for $i=0,2,4,6$ and zero otherwise.

Theorem 5. We have

$$
H^{i}\left(\pi_{0} \otimes F^{*}\right)= \begin{cases}\mathbb{C}, & i=2,4, \\ 0, & i=0,1,3,5,6,\end{cases}
$$

and

$$
H^{j}\left(\pi_{1,0} \otimes F^{*}\right)=H^{j}\left(\pi_{0,1} \otimes F^{*}\right)= \begin{cases}\mathbb{C}, & j=1,3,5, \\ 0, & j=0,2,4,6 .\end{cases}
$$

Proof. Let $\mathfrak{g}=\mathfrak{k} \oplus \mathfrak{p}$ be the Cartan decomposition of $\mathfrak{g}$. Since $\pi_{0}$ is unitary, we have

$$
H^{i}\left(\pi_{0} \otimes F^{*}\right)=\operatorname{Hom}_{K}\left(\Lambda^{i} \mathfrak{p}, \pi_{0} \otimes F^{*}\right) \cong \operatorname{Hom}_{K}\left(\Lambda^{i} \mathfrak{p} \otimes F, \pi_{0}\right) .
$$

See $[\mathbf{B W}]$. 
From a direct computation, we find that the highest weights of the $K$ types of $\Lambda^{i} \mathfrak{p}$ are given in Table 2 (with multiplicity-one).

\begin{tabular}{|c|c|}
\hline module & highest weights of $K$-types \\
\hline$\Lambda^{0} \mathfrak{p}$ & $(0,0)$ \\
\hline$\Lambda^{1} \mathfrak{p}$ & $(2,0),(0,-2)$ \\
\hline$\Lambda^{2} \mathfrak{p}$ & $(3,1),(2,-2),(1,-1),(0,0),(-1,-3)$, \\
\hline$\Lambda^{3} \mathfrak{p}$ & $(3,3),(3,-1),(2,0),(1,1),(1,-3),(0,-2),(-1,-1) ;(-3,-3)$ \\
\hline
\end{tabular}

Table 2.

For $4 \leq i \leq 6$, we have $\Lambda^{i} \mathfrak{p} \cong \Lambda^{6-i} \mathfrak{p}$ as $K$ modules.

By examining the $K$-types in $\pi_{0}$ and $F$, it is easy to see that for $i=2$ or 4 , the only common $K$-types of $\Lambda^{i} \mathfrak{p} \otimes F$ and $\pi_{0}$ is $\left(\frac{m}{2}-1,-\frac{m}{2}+1\right)$, and for all other $i, \Lambda^{i} \mathfrak{p} \otimes F$ and $\pi_{0}$ have no common $K$-types. It follows that

$$
H^{i}\left(\pi_{0} \otimes F^{*}\right) \cong \operatorname{Hom}_{K}\left(\Lambda^{i} \mathfrak{p} \otimes F, \pi_{0}\right)= \begin{cases}\mathbb{C}, & i=2,4, \\ 0, & \text { otherwise }\end{cases}
$$

Next we compute the cohomology of $\pi_{1,0} \oplus \pi_{0,1}$. Let $R$ be the second term of the socle series of $I^{+}$, namely the submodule of $I^{+}$such that $R / \pi_{0}$ is the direct sum of irreducible submodules of $I^{+} / \pi_{0}$. Thus $R$ has three irreducible constituents $\pi_{0}, \pi_{0,1}$ and $\pi_{1,0}$. We have the short exact sequence

$$
0 \rightarrow R \rightarrow I^{+} \rightarrow F \rightarrow 0 .
$$

This induces a short exact sequence

$$
0 \rightarrow R \otimes F^{*} \rightarrow I^{+} \otimes F^{*} \rightarrow F \otimes F^{*} \rightarrow 0,
$$

and the corresponding long exact sequence

$$
H^{i-1}\left(I^{+} \otimes F^{*}\right) \rightarrow H^{i-1}\left(F \otimes F^{*}\right) \rightarrow H^{i}\left(R \otimes F^{*}\right) \rightarrow H^{i}\left(I^{+} \otimes F^{*}\right) .
$$

By Proposition $2, H^{i}\left(I^{+} \otimes F^{*}\right)=0$ for $i \leq 4$. Hence

$$
H^{i}\left(R \otimes F^{*}\right) \cong H^{i-1}\left(F \otimes F^{*}\right), \quad i \leq 4 .
$$

Combining the above with a simple diagram chasing, we conclude

$$
H^{i}\left(R \otimes F^{*}\right)= \begin{cases}\mathbb{C}, & i=1,3, \\ \mathbb{C}^{2}, & i=5, \\ 0, & i=0,2,4,6 .\end{cases}
$$


We now look at the short exact sequence

$$
0 \rightarrow \pi_{0} \rightarrow R \rightarrow \pi_{1,0} \oplus \pi_{0,1} \rightarrow 0 .
$$

Applying the long exact sequence induced by the above short exact sequence as before and with another straight-forward diagram chasing, we obtain

$$
H^{j}\left(\mathfrak{g}, K ;\left(\pi_{1,0} \oplus \pi_{0,1}\right) \otimes F^{*}\right)= \begin{cases}\mathbb{C}^{2}, & j=1,3,5, \\ 0, & j=0,2,4,6 .\end{cases}
$$

Note that $F^{*} \cong F$. We now use Proposition 4 to construct an isomorphism $S$ between $\operatorname{Hom}_{K}\left(\Lambda^{j} \mathfrak{p}, \pi_{1,0} \otimes F\right)$ and $\operatorname{Hom}_{K}\left(\Lambda^{j} \mathfrak{p}, \pi_{0,1} \otimes F\right)$, which form the cochain complexes defining the $(\mathfrak{g}, K)$-cohomology groups of $\pi_{1,0} \otimes F$ and $\pi_{0,1} \otimes F$, as follows. For each $0 \leq j \leq 6$, let $D_{j}: \Lambda^{j} \mathfrak{p} \rightarrow \Lambda^{j} \mathfrak{p}$ be the map given by

$$
D_{j}\left(p_{1} \wedge \cdots \wedge p_{j}\right)=\tau\left(p_{1}\right) \wedge \cdots \wedge \tau\left(p_{j}\right) .
$$

Then for $X \in \mathfrak{k}$ and $u \in \Lambda^{j} \mathfrak{p}$,

$$
D_{j}(X u)=\tau(X) D_{j}(u) .
$$

Now for each $L \in \operatorname{Hom}_{K}\left(\Lambda^{j} \mathfrak{p}, \pi_{1,0} \otimes F\right)$, we let

$$
S(L)=\left(\mathcal{T}_{1} \otimes \mathcal{T}_{2}\right) L D^{-1}: \Lambda^{j} \mathfrak{p} \longrightarrow \pi_{0,1} \otimes F .
$$

Then

$$
S: \operatorname{Hom}_{K}\left(\Lambda^{j} \mathfrak{p}, \pi_{1,0} \otimes F\right) \rightarrow \operatorname{Hom}_{K}\left(\Lambda^{j} \mathfrak{p}, \pi_{0,1} \otimes F\right)
$$

is clearly a vector space isomorphism. Moreover it is easy to check that it commutes with coboundary operators. Consequently it induces an isomorphism from $H^{j}\left(\pi_{0,1} \otimes F\right) \rightarrow H^{j}\left(\pi_{1,0} \otimes F\right)$. Our conclusion on the cohomology of $\pi_{0,1}$ and $\pi_{1,0}$ then follows.

Remark. When $m=6$, one checks that

$$
\operatorname{dim} \operatorname{Hom}_{K}\left(\Lambda^{i} \mathfrak{p} \otimes F, \pi_{1,0}\right)=\operatorname{dim} \operatorname{Hom}_{K}\left(\Lambda^{i} \mathfrak{p} \otimes F, \pi_{0,1}\right)=1
$$

for $i=1,3,5$. Thus every common $K$-type in $\Lambda^{i} \mathfrak{p} \otimes F$ and $\pi_{1,0}$ (resp. $\pi_{0,1}$ ) contributes to the cohomology, which resembles the case of unitary representations. When $m=4 k+2$ with $k \geq 2$, the dimension of the common $K$-types in $\Lambda_{\mathfrak{p}}^{i} \otimes F$ and $\pi_{1,0}$ (resp. $\pi_{0,1}$ ) exceeds one for $i=1,3,5$. It is likely that the non-zero cohomology class in this case is represented by a combinatorially complicated expression involving various common $K$-types.

Case 2. $m \equiv 0(\bmod 4)$. In this case, $I^{+}$has two irreducible submodules $\rho_{1,0}$ and $\rho_{0,1}$ which are unitary lowest weight and highest weight modules, respectively. The lowest weight of $\rho_{1,0}$ is $\left(\frac{m}{2}, \frac{m}{2}\right)$, and the highest weight of $\rho_{0,1}$ is $\left(-\frac{m}{2},-\frac{m}{2}\right)$. Thus they are discrete series representations when $m \geq 6$. The quotient of $I^{+}$by the direct sum of $\rho_{1,0}$ and $\rho_{0,1}$ is an infinitedimensional irreducible representation $\rho_{2}$, which is non-unitary. The module diagram in this case is given in Figure 2. 


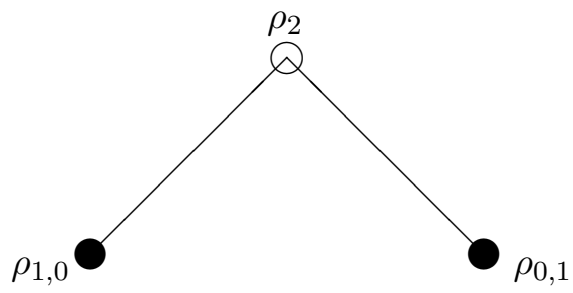

Figure 2.

Proposition 6 ([LZ2]). We have

$$
\theta^{m, 0} \cong \rho_{1,0}, \quad \theta^{0, m} \cong \rho_{0,1} .
$$

Moreover if $p, q$ are both even and $p, q \geq 2$, then $\theta^{p, q} \cong \rho_{2}$.

Since $\rho_{1,0}$ and $\rho_{0,1}$ are discrete series representations, we have $[\mathbf{B W}]$

$$
H^{i}\left(\rho_{1,0} \otimes F^{*}\right)=H^{i}\left(\rho_{0,1} \otimes F^{*}\right)= \begin{cases}\mathbb{C}, & i=3, \\ 0, & i=0,1,2,4,5,6 .\end{cases}
$$

Theorem 7. We have

$$
H^{i}\left(\rho_{2} \otimes F^{*}\right)= \begin{cases}\mathbb{C}, & i=2,4 \\ 0, & i=0,1,3,5,6 .\end{cases}
$$

Proof. We have the short exact sequence

$$
0 \rightarrow \rho_{1,0} \oplus \rho_{0,1} \rightarrow I^{+} \rightarrow \rho_{2} \rightarrow 0 .
$$

This induces a long exact sequence

$$
\begin{aligned}
H^{i}\left(\left(\rho_{1,0} \oplus \rho_{0,1}\right) \otimes F^{*}\right) \rightarrow H^{i}\left(I^{+} \otimes F^{*}\right) & \rightarrow H^{i}\left(\rho_{2} \otimes F^{*}\right) \\
& \rightarrow H^{i+1}\left(\left(\rho_{1,0} \oplus \rho_{0,1}\right) \otimes F^{*}\right) .
\end{aligned}
$$

We then conclude that

$$
H^{i}\left(\rho_{2} \otimes F^{*}\right) \cong H^{i}\left(I^{+} \otimes F^{*}\right), \quad i=0,1,4,5,6 .
$$

Combining this with the result on the cohomology of $I^{+}$, and the Poincare duality for the cohomology of an irreducible admissible (g, $K)$-module $([\mathbf{B W}])$, we obtain the desired result.

Finally we shall prove Proposition 4 . We shall construct a vector space isomorphism $\mathcal{T}: I^{+} \rightarrow I^{+}$and an automorphism $\tau$ of $\mathfrak{s p}(4, \mathbb{C})$ such that

$$
\mathcal{T}(X v)=\tau(X) \mathcal{T}(v), \quad\left(X \in \mathfrak{s p}(4, \mathbb{C}), v \in I^{+}\right),
$$

$\mathcal{T}\left(\pi_{1,0}\right)=\pi_{0,1}$ and $\mathcal{T}(F)=F$. Hence the restriction of $\mathcal{T}$ to $\pi_{1,0}$ and $F$ give the maps $\mathcal{T}_{1}$ and $\mathcal{T}_{2}$ in Proposition 4. 
Let

$$
c=\frac{1}{\sqrt{2}}\left(\begin{array}{cc}
I_{2} & i I_{2} \\
i I_{2} & I_{2}
\end{array}\right) .
$$

We consider the automorphisms $\psi_{1}$ and $\psi_{2}$ of $\mathfrak{s p}(4, \mathbb{C})$ given by

$$
\psi_{1}(X)=c^{-1} X c,
$$

and

$$
\psi_{2}(X)=\left(\begin{array}{cccc}
-d & -b & -w & -v \\
-c & -a & -v & -u \\
-z & -y & d & c \\
-y & -x & b & a
\end{array}\right)
$$

where

$$
X=\left(\begin{array}{cccc}
a & b & x & y \\
c & d & y & z \\
u & v & -a & -c \\
v & w & -b & -d
\end{array}\right) \in \mathfrak{s p}(4, \mathbb{C})
$$

Let $\tau=\psi_{1}^{-1} \circ \psi_{2} \circ \psi_{1}$. Then $\tau$ is an automorphism of $\mathfrak{s p}(4, \mathbb{C})$ of order 2 .

The complexification of the Lie algebra of $K$ is given by

$$
\mathfrak{k}=\left\{\left(\begin{array}{cc}
A & B \\
-B & A
\end{array}\right): A, B \in \mathfrak{g l}_{2}(\mathbb{C}), A=-A^{t}, B=B^{t}\right\} .
$$

We have

$$
\mathfrak{k} \cong \mathfrak{g l}_{2}(\mathbb{C})
$$

and

$$
\psi_{1}(\mathfrak{k})=\left\{\left(\begin{array}{cc}
A & 0 \\
0 & -A^{t}
\end{array}\right): A \in \mathfrak{g l}_{2}(\mathbb{C})\right\}
$$

Recall that $I^{+}$has the following $K$-type decomposition:

$$
\left.I^{+}\right|_{K} \cong \sum_{\lambda \in \Lambda_{e}^{+}(2)} V_{\lambda},
$$

where $V_{\lambda}$ denotes a copy of the irreducible representation of $K$ with highest weight $\lambda$. Let $\xi_{\lambda}$ be the highest weight vector in $V_{\lambda}$ given in Lemma 2.1 of [Le2]. 
Note that the restriction of $\psi_{2}$ to $\psi_{1}(\mathfrak{k})$ is given by

$$
\psi_{2}\left(\begin{array}{cccc}
a & b & 0 & 0 \\
c & d & 0 & 0 \\
0 & 0 & -a & -c \\
0 & 0 & -b & -d
\end{array}\right)=\left(\begin{array}{cccc}
-d & -b & 0 & 0 \\
-c & -a & 0 & 0 \\
0 & 0 & d & c \\
0 & 0 & b & a
\end{array}\right),
$$

which corresponds to the automorphism of $\mathfrak{g l}_{2}(\mathbb{C})$ given in [Le1, p. 318]. Hence for any $\lambda \in \Lambda_{e}^{+}(2)$, there is a unique vector space isomorphism $T_{\lambda}$ : $V_{\lambda} \rightarrow V_{\lambda^{*}}$ such that:

(i) $T_{\lambda}\left(\xi_{\lambda}\right)=\xi_{\lambda^{*}}$,

(ii) $T_{\lambda}(X v)=\tau(X) T_{\lambda}(v), \forall X \in \mathfrak{k}, \forall v \in \pi_{1,0}$.

Here $\lambda^{*}=\left(-\lambda_{2},-\lambda_{1}\right)$ if $\lambda=\left(\lambda_{1}, \lambda_{2}\right)$. Thus $V_{\lambda^{*}}$ is the contragradient to $V_{\lambda}$ as a $K$ module.

We now let

$$
\mathcal{T}=\oplus_{\lambda \in \Lambda_{e}^{+}(2)} T_{\lambda} .
$$

Then $\mathcal{T}: I^{+} \rightarrow I^{+}$is a vector space isomorphism such that $\mathcal{T}\left(\pi_{1,0}\right)=\pi_{0,1}$ and $\mathcal{T}(F)=F$. We shall show that

$$
\mathcal{T}(X v)=\tau(X) \mathcal{T}(v) \quad \forall X \in \mathfrak{s p}(4, \mathbb{C}), \forall v \in I^{+} .
$$

Let $\mathfrak{s p}(4, \mathbb{C})=\mathfrak{k} \oplus \mathfrak{p}^{+} \oplus \mathfrak{p}^{-}$be the Harish-Chandra decomposition, where

$$
\begin{gathered}
\mathfrak{p}^{+}=\left\{\left(\begin{array}{cc}
B & i B \\
i B & -B
\end{array}\right): B \in \mathfrak{g l}_{2}(\mathbb{C}), B=B^{t}\right\}, \\
\mathfrak{p}^{-}=\left\{\left(\begin{array}{cc}
B & -i B \\
-i B & -B
\end{array}\right): B \in \mathfrak{g l}_{2}(\mathbb{C}), B=B^{t}\right\} .
\end{gathered}
$$

It suffices to prove that Eq. (3) holds for all $X=p \in \mathfrak{p}^{+}$or $\mathfrak{p}^{-}$. We fix a $K$-type $V_{\lambda}$ in $I^{+}$and we let $\varphi: \mathfrak{p}^{+} \otimes V_{\lambda} \rightarrow \mathfrak{p}^{-} \otimes V_{\lambda^{*}}$ be the vector space isomorphism given by

$$
\varphi(p \otimes v)=\tau(p) \otimes \mathcal{T}(v), \quad \forall p \in \mathfrak{p}^{+}, v \in V_{\lambda} .
$$

Then one can check that

$$
\varphi[X(p \otimes v)]=\tau(X) \varphi(p \otimes v), \quad \forall X \in \mathfrak{k}, p \in \mathfrak{p}^{+}, v \in V_{\lambda} .
$$

For $j=1,2$, let $\nu_{\lambda+2 e_{j}}$ (resp. $\omega_{\lambda^{*}-2 e_{j}}$ ) be the highest weight vector in $\mathfrak{p}^{+} \otimes V_{\lambda}$ (resp. $\left.\mathfrak{p}^{-} \otimes V_{\lambda^{*}}\right)$ of weight $\lambda+2 e_{j}$ (resp. $\lambda^{*}-2 e_{j}$ ) as given in part (a) of Proposition 3.1 of [Le2]. It is easy to verify that for $j=1,2$,

$$
\varphi\left(\nu_{\lambda+2 e_{j}}\right)=\omega_{\left(\lambda+2 e_{j}\right)^{*}}=\omega_{\lambda^{*}-2 e_{3-j}} .
$$


We now fix $p \in \mathfrak{p}^{+}$and $v \in V_{\lambda}$. Denote by $\mathfrak{U}(\mathfrak{k})$ the universal enveloping algebra of $\mathfrak{k}$. Then there are elements $X_{1}, X_{2}, X_{3} \in \mathfrak{U}(\mathfrak{k})$ such that

$$
p \otimes v=X_{1} \nu_{\lambda+2 e_{1}}+X_{2} \nu_{\lambda+2 e_{2}}+X_{3} \nu_{\lambda+e_{1}+e_{2}},
$$

where $\nu_{e_{1}+e_{2}}$ is a highest weight vector in $\mathfrak{p}^{+} \otimes V_{\lambda}$ of weight $\lambda+e_{1}+e_{2}$. Then $\varphi(p \otimes v)=\tau(p) \otimes \mathcal{T}(v)=\left(\tau X_{1}\right) \omega_{\lambda^{*}-2 e_{2}}+\left(\tau X_{2}\right) \omega_{\lambda^{*}-2 e_{1}}+\left(\tau X_{3}\right) \omega_{\lambda^{*}-e_{1}-e_{2}}$, where $\omega_{\lambda-e_{1}-e_{2}}=\varphi\left(\nu_{\lambda+e_{1}+e_{2}}\right)$ is a highest weight vector in $\mathfrak{p}^{-} \otimes V_{\lambda^{*}}$ of weight $\lambda^{*}-e_{1}-e_{2}$. Recall that we have $\mathfrak{k}$-maps $m_{\lambda}: \mathfrak{p}^{+} \otimes V_{\lambda} \rightarrow \pi_{1,0}$ and $m_{\lambda^{*}}: \mathfrak{p}^{-} \otimes V_{\lambda^{*}} \rightarrow \pi_{0,1}$ given by

$$
\begin{aligned}
m_{\lambda}(p \otimes v) & =p v, & & \left(p \in \mathfrak{p}^{+}, v \in V_{\lambda}\right), \\
m_{\lambda^{*}}(q \otimes u) & =q u, & & \left(q \in \mathfrak{p}^{-}, u \in V_{\lambda^{*}}\right) .
\end{aligned}
$$

Now using Eq. (4),

$$
\begin{aligned}
p v & =m_{\lambda}(p \otimes v) \\
& =X_{1} m_{\lambda}\left(\nu_{\lambda+2 e_{1}}\right)+X_{2} m_{\lambda}\left(\nu_{\lambda+2 e_{2}}\right)+X_{3} m_{\lambda}\left(\nu_{\lambda+e_{1}+e_{2}}\right) \\
& =\left(\alpha-\lambda_{1}\right) X_{1} \xi_{\lambda+2 e_{1}}+\left(\alpha-\lambda_{2}+1\right)\left(\lambda_{1}-\lambda_{2}\right) X_{2} \xi_{\lambda+2 e_{2}},
\end{aligned}
$$

by Proposition 3.7 of [Le2], where $\alpha=-m / 2$. Applying the map $\mathcal{T}$ to this equation gives

(7) $\mathcal{T}(p v)=\left(\alpha-\lambda_{1}\right)\left(\tau X_{1}\right) \xi_{\lambda^{*}-2 e_{2}}+\left(\alpha-\lambda_{2}+1\right)\left(\lambda_{1}-\lambda_{2}\right)\left(\tau X_{2}\right) \xi_{\lambda^{*}-2 e_{1}}$.

On the other hand, by applying the map $m_{\lambda^{*}}$ to Eq. (5), we obtain

$$
\begin{aligned}
& \tau(p) \mathcal{T}(v) \\
&=m_{\lambda^{*}}[\tau(p) \otimes \mathcal{T}(v)] \\
&=m_{\lambda^{*}}(\varphi(p \otimes v)) \\
&=\left(\tau X_{1}\right) m_{\lambda^{*}}\left(\omega_{\lambda^{*}-2 e_{2}}\right)+\left(\tau X_{2}\right) m_{\lambda^{*}}\left(\omega_{\lambda^{*}-2 e_{1}}\right)+\left(\tau X_{3}\right) m_{\lambda^{*}}\left(\omega_{\lambda^{*}-e_{1}-e_{2}}\right) \\
&=\left[\alpha+\left(-\lambda_{1}\right)+2-2\right]\left(\tau X_{1}\right) \xi_{\lambda^{*}-2 e_{2}} \\
& \quad+\left(\alpha-\lambda_{2}+1\right)\left[-\lambda_{2}-\left(-\lambda_{1}\right)\right]\left(\tau X_{2}\right) \xi_{\lambda^{*}-2 e_{1}} \\
&=\left(\alpha-\lambda_{1}\right)\left(\tau X_{1}\right) \xi_{\lambda^{*}-2 e_{2}}+\left(\alpha-\lambda_{2}+1\right)\left(\lambda_{1}-\lambda_{2}\right)\left(\tau X_{2}\right) \xi_{\lambda^{*}-2 e_{1}} \\
&= \mathcal{T}(p v),
\end{aligned}
$$

by Eq. (7). The proof for

$$
\mathcal{T}(p v)=\tau(p) \mathcal{T}(v), \quad\left(p \in \mathfrak{p}^{-}, v \in I^{+}\right)
$$

is similar. 


\section{References}

[Ad] J. Adams, Discrete spectrum of the reductive dual pair $(O(p, q), S p(2 m))$, Invent. Math., 74 (1983), 449-475.

[An] G. Anderson, Theta functions and holomorphic differential forms on compact quotients of bounded symmetric domains, Duke Math. J., 50(4) (1983), 1137-1170.

[BW] A. Borel and N. Wallach, Continuous cohomology, discrete subgroups, and representations of reductive groups, Ann. of Math. Stud., 94, Princeton Univ. Press, Princeton, 1980.

[BT] T. Brocker and T. Dieck, Representations of Compact Lie Groups, Graduate Texts in Mathematics, Vol. 98, Springer-Verlag, New York, 1985.

[HK] M. Harris and S. Kudla, Arithmetic automorphic froms for the nonholomorphic discrete series of $G S p(2)$, Duke Math. J., 66 (1992), 59-121.

[H1] R. Howe, Remarks on classical invariant theory, Trans. Amer. Math. Soc., 313 (1989), 539-570.

[H2] - Transcending classical invariant theory, J. Amer. Math. Soc., 2 (1989), 535-552.

[H3],$\theta$-series and invariant theory, in "Automorphic forms, representations and L-functions," Proc. Sympos. Pure Math., 33(1), 275-286, Amer. Math. Soc., Providence, RI, 1979.

[H4] _ Perspectives on invariant theory: Schur duality, multiplicity-free actions and beyond, Israel Mathematical Conference Proceedings.

[KV] M. Kashiwara and M. Vergne, On the Segal-Shale-Weil representations and harmonic polynomials, Invent. Math., 44 (1978), 1-47.

[Ka] D. Kazhdan, Some applications of the Weil representations, Jour. D'Analyse Math., 32 (1977), 235-248.

[Ko] B. Kostant, Lie algebra cohomology and the generalized Borel-Weil theorem, Annals of Math., 74(2) (1961), 329-387.

[KR1] S. Kudla and S. Rallis, Degenerate principal series and invariant distributions, Israel J. Math., 69 (1990), 25-45.

[KR2] — A regularized Siegel-Weil formula: The first term identity, Annals of Math., 140 (1994), 1-80.

[Le1] S. Lee, On some degenerate principal series representations of $U(n, n)$, J. Funct. Anal., 126 (1994), 305-366.

[Le2] - Degenerate principal series representation of $\operatorname{Sp}(2 n, \mathbb{R})$, Compositio Math., 103 (1996), 123-151.

[LZ1] S. Lee and C-B. Zhu, Degenerate principal series and local theta correspondence, Trans. A.M.S., 350(12) (1998), 5017-5046.

[LZ2] _ Degenerate principal series and local theta correspondence II, Israel J. Math., 100 (1997), 29-59.

[Li1] J-S. Li, Singular unitary representations of classical groups, Invent. Math., 97 (1989), 237-255.

[Li2] - Theta liftings for unitary representations with non-zero cohomology, Duke Math. J., 61 (1990), 913-937. 
[Li3] Non-vanishing theorems for the cohomology of certain arithmetic quotients, J. Reine Angew. Math., 428 (1992), 177-217.

[P] T. Przebinda, The duality correspondence of infinitesimal characters, Colloq. Math., 70(1) (1996), 93-102.

[RS] S. Rallis and G. Schiffmann, Weil representation I. Intertwining distributions and discrete spectrum, Memoirs of the A.M.S., 25(231) (1980), 1-203.

[S] S. Sahi, Explicit Hilbert spaces for certain unipotent representations, Invent. Math., 110 (1992), 409-418.

[VZ] D. Vogan and G. Zuckerman, Unitary representations with non-zero cohomology, Compositio Math., 53 (1984), 51-90.

[W] N. Wallach, Square integrable automorphic forms and cohomology of arithmetic quotients of $S U(p, q)$, Math. Annalen, 266 (1984), 261-278.

[Z] G. Zuckerman, Continuous cohomology and unitary representations of real reductive groups, Annals of Math., 107(2) (1978), 495-516.

Received April 27, 1998 and revised February 1, 2000.

Department of Mathematics

National University of Singapore

10 Kent Ridge Crescent

SINGAPORE 119260

Republic of Singapore

E-mail address: matleest@nus.edu.sg

Department of Mathematics

NATional University OF Singapore

10 Kent Ridge Crescent

SINGAPORE 119260

Republic of Singapore

E-mail address: matzhucb@nus.edu.sg 